\title{
Bean Preferences Vary by Acculturation Level among Latinas and by Ethnicity with Non-Hispanic White Women
}

\author{
Michelle M. Heer and Donna M. Winham * (D) \\ Food Science \& Human Nutrition, Iowa State University, Ames, IA 50010, USA; mmheer@gmail.com \\ * Correspondence: dwinham@iastate.edu; Tel.: +1-515-294-5040
}

Received: 5 March 2020; Accepted: 20 March 2020; Published: 22 March 2020

check for updates

\begin{abstract}
With high levels of protein, fiber, folate, iron and other micronutrients, the Dietary Guidelines for Americans recommends eating beans for optimal nutrition. Low-income women are at greater risk of nutrition-related health disparities. Use of beans may change among Hispanic women (Latinas) during acculturation, but few studies exist that describe specific preferences of this important traditional food. Preserving or promoting beans in the diets of all low-income women could improve dietary quality. The study objectives were to describe consumption frequency, purchasing patterns, and attitudes toward dry and canned beans, by acculturation level among Latinas and by ethnicity with non-Hispanic White women. Survey data were collected from 356 women $(\mu 32$ y \pm 9 ; $81 \%$ Latina), who were enrolled in, or eligible for, a federal nutrition assistance, or unemployment, program in Phoenix, Arizona, USA. Participants had positive attitudes toward beans overall. Less acculturated and bicultural Latinas bought dry beans more often than their peers. Price was considered important in canned bean selection for Non-Hispanic White women, and less acculturated Latinas had poorer attitudes toward canned. Awareness of these attitudes and preferred traits of low-income women suggests ways to message populations to maintain or increase bean consumption. Negative views of canned beans by Latinas should be investigated further. Inclusion of canned beans in nutrition assistance programs may benefit those unfamiliar with preparing dry beans.
\end{abstract}

Keywords: pulses; food security; canned foods; Mexican Americans; dietary acculturation; consumer preferences; immigrants; health disparities; nutrition knowledge

\section{Introduction}

Beans have been a central element of Mexican and Central American cuisine since pre-Hispanic times [1], and annual per-capita dry-bean consumption is nearly four times greater in Mexico (11 kg) than in the United States (US) (3 kg) [2]. When people from Latin American countries immigrate to the US, their consumption of beans and other traditional foods may decline over time with acculturation and adaptation to new socio-ecological environments [3-5]. Due to variability in methodology, Latino subgrouping, and geographic location, dietary acculturation research has yielded conflicting results, although some constant dietary changes have been identified [3]. Consistent alterations with time include increased intake of sugar-sweetened beverages and fast foods and decreased consumption of fruits, rice, and beans [3], all of which can elevate chronic disease risk, especially when coupled with poverty and race-related health inequities associated with Latinos in the US [6-8]. The US government demographic classification 'Hispanic' refers to people with origins in a Spanish-speaking country regardless of personal cultural affiliation or language [9]. In this research, 'Latinas' describes women who retain some degree of Latin American cultural practices and ethnic identity. By definition, the termHispanic is used in the context of government statistics and describes ancestral origin, not necessarily cultural affiliation [9]. 
Inclusion of beans in the diets of all population groups, including Hispanic immigrants, is a positive approach which can improve dietary quality, increase vegetable intake and lower chronic-disease risk [5,7]. Beans are noted for their high fiber, protein, folate, iron, potassium, and magnesium content $[7,10,11]$. Except for protein, these essential nutrients are lacking in the diets of many Americans [10,11]. National survey data show that, on any given day, less than $8 \%$ of all US adults, compared to $25 \%$ of Hispanic Americans, consume pulses [7,10]. Pulses, defined as the edible seeds from mature pods, include dry beans, peas, lentils, chickpeas, and black-eyed peas. "Dry beans" refers to bean seeds both dried and bagged, and those cooked and canned, but does not include green or string beans eaten as pods [7,11]. Popular dry bean varieties in the US are pinto, black, navy, white, and kidney [11]. Pulse production also contributes to sustainable agriculture and improved nutrition globally [12].

The 2015 Dietary Guidelines for Americans (DGA) mention consuming pulses for dietary quality and better health [10]. The Special Supplemental Food Program for Women, Infants and Children (WIC) includes them in their food packages, $[13,14]$ and the Expanded Food and Nutrition Education Program (EFNEP) curriculum features pulses in its national lesson plan as alternative protein choices [14]. These programs focus on reducing disparities in food security and improving nutrition knowledge, primarily for low-income and at-risk women with children [14]. Promoting canned beans as well as dry is a way to reduce meal preparation time and increase access to these healthy foods [13,15].

Important factors in reducing health disparities among Latinas may include identifying preferences for bean purchases (type, form, product size, origin), attitudes toward bean consumption, and degree of acculturation [5,15]. Understanding the preferences and attitudes of all low-income women can provide additional insight into overall family consumption patterns $[16,17]$. While private companies may have marketing research data, this information is not publicly available and may represent views of consumers who are not low-income. Few studies have investigated the attitudes or purchasing preferences of low-income women, specifically Latinas, regarding beans or the other foods obtained through food-assistance programs $[15,16,18,19]$. This paucity of detail interferes with the capability of nutritionists and health professionals to effectively promote pulse consumption $[15,20]$. Nutrition education programs that account for women's established opinions can leverage beneficial behaviors and dispel myths and misconceptions through tailored messages [21]. Using cultural rituals in nutrition programming can also increase the appeal of traditional foods [22].

While about $16 \%$ of the US population in 2010 was Hispanic, they comprised about $30 \%$ of Arizona residents [23]. In Maricopa county, roughly $66 \%$ of female Arizona WIC clients and 52\% of Maricopa county EFNEP participants self-reported as Hispanic in 2011, when these data were collected [24,25]. To address research gaps on factors that influence bean consumption, such as acculturation level, ethnicity, preferred dry and canned bean purchase characteristics, knowledge and attitudes, and to help guide service improvements of related Arizona agencies, e.g., EFNEP, a survey was administered to low-income Hispanic and non-Hispanic White (NHW) women in metropolitan Phoenix, Arizona. The survey's objectives were to: 1 ) compare consumption frequencies; 2 ) assess purchasing preferences; and 3) describe attitudes toward beans, in general and to canned beans, in terms of acculturation levels and ethnicity among low-income Latinas and non-Hispanic White women. It was hypothesized that dry and canned bean consumption, purchasing preferences, and attitude scores would vary by acculturation status and ethnicity.

\section{Materials and Methods}

\subsection{Participants and Survey Development}

Women aged 18-55 years who were currently enrolled or eligible to participate in a metropolitan Phoenix federal assistance program (WIC, EFNEP, Temporary Aid for Needy Families, or SNAP), were recruited between May and December 2011. Data collection was conducted at EFNEP classes, at a WIC clinic with a high percentage of Hispanic clientele and an unemployment job center. 
The survey was developed based on a literature review, previous research [26], two focus groups with EFNEP participants $(n=24)$, and one focus group with extension instructional specialists $(n=7)$ who were laypersons from the communities served. Focus groups were moderated by the co-author (D.M.W.) and a bilingual/bicultural EFNEP research staff member. Two EFNEP participants preferred to respond in Spanish, but had some English fluency. Audio recordings were transcribed by the collaborating EFNEP researcher. Topics discussed were views of beans, cooking practices, role of beans in traditional diets, preference for certain bean characteristics based on culture, cultural acceptability, and perceived barriers and motivators to bean consumption. Seven Likert-type general-bean questions related to perceptions about taste, association of beans with poor people, if friends eat beans, issues with gas, and ease of preparation of dry beans, were asked. A second set of seven Likert-type questions related to taste, cultural and family acceptance, nutrition, and cost of canned beans were also asked. Response categories for these statements were: strongly disagree, disagree, neutral, agree, and strongly agree.

Eight public health nutrition researchers and three EFNEP field staff evaluated the semi-final survey instrument for content validity. All bean-related questions were translated by a bilingual/bicultural staff member, then back-translated by an external consultant. A pilot test with three different EFNEP classes $(n=23)$ allowed participants to provide feedback and discuss points of confusion or meaning on the questions in general, and the Spanish wording. The co-author and the EFNEP bilingual researcher led these sessions also. Pilot test participant data were excluded from analysis. Before official data collection began, minor format and word changes were made based on pilot input. Survey findings regarding the knowledge of bean-related health benefits, utilization of food assistance programs, and health behaviors have been previously reported [27-29].

Acculturation status was evaluated by the Bidimensional Acculturation Scale (BAS) [30]. Latinas were classified into three groups: Hispanic-dominant (less acculturated), bicultural, and English-dominant (more acculturated) [30]. Non-Hispanic White women (NHW) were their own category. For questions on demographics, ethnicity, race, household characteristics, and food costs, the standard EFNEP enrollment form was used [31]. Respondents completed the USDA Core Food Security Module to measure food security [32,33]. The validated English and Spanish versions of these two instruments were used. Participants were read a verbal script in English or Spanish prior to receiving the survey whose completion was considered informed consent. No personal identification information was obtained from participants. The Arizona State University Institutional Review Board deemed the study exempt.

\subsection{Survey Administration and Data Collection}

Self-administration of the surveys occurred at the end of the third or fourth session of the standard EFNEP six-class series before beans and other pulses were specifically discussed. Bilingual EFNEP instructional specialists were available to aid participants with form completion. Small incentives, e.g., grocery coupons, writing pads, pens, totes, etc., were given at survey completion. Two to four staff personnel, including at least one bilingual staff person, set up a desk, placed information signs, and distributed information to waiting clients at the WIC clinic and the unemployment center. Individuals completing the survey, at either the WIC clinic or the unemployment center, received a $\$ 3.00$ cash incentive.

\subsection{Data Transformations and Statistical Analysis}

Principal components analysis with varimax rotation of the 14 Likert statements produced four factors with eigenvalues greater than 1.0 and explained $59.3 \%$ of the variance. Factor loadings less than 0.40 were suppressed [34]. Factor 1 was general bean attitudes (eigenvalue 3.89, 27.82\% variance). Factor 2 and Factor 3 (eigenvalues 2.15, 1.25; 15.35\%, 8.93\% variance, respectively) were both canned-bean related variables. Only two items loaded on Factor 4 (eigenvalue $1.004 ; 7.17 \%$ variance). Reliability analysis resulted in a five-item general-bean scale (Cronbach's alpha $=0.78)$ from Factor 1 , and a 
six-item canned-bean scale (Cronbach's alpha $=0.76)$ as a combination of items in Factor 2 and 3 [34]. The Likert-type responses were analyzed in their original five categories using Chi-square and ANOVA by ethnicity and acculturation level. For clarity of display, responses were condensed into 3-categories of 'disagree, neutral, agree' in the results section. A general linear model representing acculturation level, age, education, household size, food security score and bean-consumption frequency was included in predictive models of general-bean and canned-bean attitude scale scores. Data analysis utilized SPSS V. 25 (IBM, Armonk, NY, USA) software, with a significance level of $p<0.050$.

\section{Results}

The completion rate for eligible women was $90 \%$ (356/397) with $44 \%$ of the total sample recruited from WIC, 42\% from EFNEP, and 14\% from the job center. The majority of the 356 participants self-identified as Hispanic (81\%), with 91\% of these stating Mexican or Mexican American ancestry, and $66 \%$ choosing to complete the survey in Spanish. Ninety-seven percent of the less acculturated and $62 \%$ of the bicultural Latinas were born outside the US, whereas $95 \%$ of the more acculturated Latinas and $98.7 \%$ of NHW women were US-born $(p<0.001)$. Less acculturated Latinas had fewer years of education, larger household size, more children, and a higher percentage were married in comparison to their peers (all $p<0.001$; Table 1). More acculturated Latinas were significantly younger $(p<0.001)$, and more likely to be unmarried than their peers $(p<0.001)$. The less acculturated Latinas had the highest percentage in the 'low' food security category, but the more acculturated Latinas had the largest percentage reporting 'very low' food security $(p=0.008)$.

Table 1. Distribution of demographic and household characteristics of low-income Arizona women by Bidimensional Acculturation Scale classifications $(N=356)$.

\begin{tabular}{|c|c|c|c|c|c|c|}
\hline Characteristics & $\begin{array}{c}\text { Total } \\
\text { Sample }\end{array}$ & $\begin{array}{l}\text { Less Acculturated } \\
\text { Latinas } 42 \%(151)\end{array}$ & $\begin{array}{c}\text { Bicultural } \\
\text { Latinas } 25 \% \\
\quad(88)\end{array}$ & $\begin{array}{c}\text { More } \\
\text { Acculturated } \\
\text { Latinas } 12 \%(41)\end{array}$ & $\begin{array}{l}\text { Non-Hispanic } \\
\text { White } 21 \% \\
\text { (76) }\end{array}$ & $p$ Value \\
\hline \multicolumn{7}{|c|}{$\longleftrightarrow$ mean \pm standard deviation $\longrightarrow$} \\
\hline Age in years $( \pm S D)$ & $33.1 \pm 9.2$ & $34.3 \pm 7.2$ & $30.6 \pm 8.9$ & $27.3 \pm 9.3$ & $36.3 \pm 10.8$ & $<0.001$ \\
\hline Number of children under age 20 & $2.3 \pm 1.3$ & $2.7 \pm 1.2$ & $2.4 \pm 1.3$ & $2.0 \pm 1.3$ & $1.5 \pm 1.4$ & $<0.001$ \\
\hline Number of adults & $2.3 \pm 1.0$ & $2.4 \pm 1.0$ & $2.5 \pm 1.0$ & $2.1 \pm 0.8$ & $2.0 \pm 0.9$ & 0.011 \\
\hline Total household size & $4.6 \pm 1.8$ & $5.1 \pm 1.6$ & $4.9 \pm 1.7$ & $4.2 \pm 1.6$ & $3.5 \pm 1.8$ & $<0.001$ \\
\hline \multicolumn{7}{|l|}{ Years of Education } \\
\hline 6 th grade or less & 11.2 & $25.2_{\mathrm{a}}$ & $2.3_{\mathrm{b}}$ & $0_{\mathrm{b}}$ & $0_{\mathrm{b}}$ & \multirow{6}{*}{$<0.001$} \\
\hline 7-9 th grade-Junior High & 15.4 & $27.2_{\mathrm{a}}$ & $11.4_{\mathrm{b}}$ & $4.9_{\mathrm{b}, \mathrm{c}}$ & $2.6_{c}$ & \\
\hline $10-11$ th grade & 14.3 & $14.6_{\mathrm{a}, \mathrm{b}}$ & $20.5_{\mathrm{b}}$ & $14.6_{\mathrm{a}, \mathrm{b}}$ & $6.6 \mathrm{a}$ & \\
\hline 12 th grade or GED & 22.8 & $21.2_{\mathrm{a}}^{\mathrm{b}}$ & $25.0_{\mathrm{a}, \mathrm{b}}$ & $39.0_{\mathrm{b}}$ & $14.5 \mathrm{a}$ & \\
\hline Some college-no degree & 24.4 & $6.6_{a}$ & $27.3_{\mathrm{b}}$ & $31.7_{\mathrm{b}}$ & $52.6_{c}$ & \\
\hline Associates degree or more & 11.8 & $5.3 \mathrm{a}$ & $13.6_{\mathrm{b}}$ & $9.8_{\mathrm{a}, \mathrm{b}}$ & $23.7_{\mathrm{b}}$ & \\
\hline \multicolumn{7}{|l|}{ Marital Status } \\
\hline Single/Divorced/Widowed & 36.1 & $17 . \mathrm{a}_{\mathrm{a}}$ & $36.4_{b}$ & $68.3_{\mathrm{c}}$ & $55.3_{\mathrm{c}}$ & \multirow[t]{2}{*}{$<0.001$} \\
\hline Married/Cohabitating & 63.9 & $82.7 \mathrm{a}$ & $63.6_{\mathrm{b}}$ & $31.7_{\mathrm{c}}$ & $44.7_{\mathrm{c}}$ & \\
\hline \multicolumn{7}{|l|}{ Food Security Status } \\
\hline High & 48.4 & $44.8 \mathrm{a}$ & $54.5 \mathrm{a}$ & $48.8_{\mathrm{a}}$ & $48.0_{\mathrm{a}}$ & \multirow{3}{*}{0.008} \\
\hline Low & 36.9 & $46.9 \mathrm{a}$ & $30.7_{\mathrm{b}}$ & $24.4_{\mathrm{b}}$ & $32.0_{\mathrm{b}}$ & \\
\hline Very low & 14.7 & $8.4_{\mathrm{a}}$ & $14.8_{\mathrm{a}, \mathrm{b}}$ & $26.8_{\mathrm{b}}$ & $20.0_{\mathrm{b}}$ & \\
\hline
\end{tabular}

Same subscript letters $(\mathrm{a}, \mathrm{b}$, etc.) indicate column proportions that are not significantly different from each other.

Higher percentages of the less acculturated and bicultural Latinas purchased beans of any type, consumed them three or more times per week, and had knowledge of dry bean preparation than the more acculturated Latinas and NHW women ( $p<0.001$ for all). All of the Latinas were more likely to have someone in their household cook dry beans at least $1-2$ times per week $(p<0.001)$ (Table 2). 
Table 2. Bean purchasing practices and preferences of low-income Arizona women by Bidimensional Acculturation Scale categories $(N=356)$.

\begin{tabular}{|c|c|c|c|c|c|c|}
\hline Characteristics & $\begin{array}{c}\text { Total } \\
\text { Sample }\end{array}$ & $\begin{array}{l}\text { Less Acculturated } \\
\text { Latinas } 42 \% \text { (151) }\end{array}$ & $\begin{array}{c}\text { Bicultural } \\
\text { Latinas } 25 \%(88)\end{array}$ & $\begin{array}{c}\text { More } \\
\text { Acculturated } \\
\text { Latinas } 12 \%(41)\end{array}$ & $\begin{array}{l}\text { Non-Hispanic } \\
\text { White } 21 \%(76)\end{array}$ & $p$ Value \\
\hline & & $\longleftarrow$ & $\%$ & $\longrightarrow$ & & \\
\hline \multicolumn{7}{|l|}{ How often do you eat beans? } \\
\hline Once a month or less & 11.0 & $4.6_{\mathrm{a}}$ & $8.0_{\mathrm{a}, \mathrm{b}}$ & $17.1_{\mathrm{b}, \mathrm{c}}$ & $23.7_{\mathrm{c}}$ & \multirow{5}{*}{$<0.001$} \\
\hline $2-3$ times per month & 25.8 & $13.2 \mathrm{a}$ & $27.3_{\mathrm{b}}$ & $34.1_{\mathrm{b}, \mathrm{c}}$ & $44.7_{\mathrm{c}}$ & \\
\hline $1-2$ times per week & 29.5 & $27.8 \mathrm{a}$ & $30.7 \mathrm{a}$ & $39.0 \mathrm{a}$ & $26.3_{\mathrm{a}}$ & \\
\hline 3-4 times per week & 22.5 & $34.1_{\mathrm{a}}$ & $23.9 \mathrm{a}$ & $4.9_{\mathrm{b}}$ & $5.3_{\mathrm{b}}$ & \\
\hline $5+$ times per week & 11.2 & $19.2 \mathrm{a}$ & $10.2_{\mathrm{a}, \mathrm{b}}$ & $4.9_{\mathrm{b}, \mathrm{c}}$ & $0_{\mathrm{c}}$ & \\
\hline $\begin{array}{l}\text { Buys any beans (dry or canned) } \\
\text { Type of bean purchased }\end{array}$ & \multicolumn{5}{|c|}{ Type of bean purchased } & $<0.001$ \\
\hline Both & 44.1 & $30.5 \mathrm{a}$ & $55.7_{\mathrm{b}}$ & $63.4_{\mathrm{b}}$ & $47.4_{\mathrm{b}}$ & \multirow{3}{*}{$<0.001$} \\
\hline Dry bean only & 42.7 & $68.2 \mathrm{a}$ & $37.5_{\mathrm{b}}$ & $22.0_{\mathrm{b}, \mathrm{c}}$ & $9.2_{c}$ & \\
\hline Canned bean only & 5.9 & $0.7 \mathrm{a}$ & $4.5_{\mathrm{b}}$ & $2.4_{a, b}$ & $19.7_{\mathrm{c}}$ & \\
\hline Neither dry or canned & 7.3 & $0.7 \mathrm{a}$ & $2.3 \mathrm{a}$ & $12.2_{\mathrm{b}}$ & $23.7_{\mathrm{b}}$ & \\
\hline Dry beans cooked in household & 90.1 & $96.7 \mathrm{a}$ & $95.5 \mathrm{a}$ & $82.9 \mathrm{~b}$ & $75.0_{\mathrm{b}}$ & $<0.001$ \\
\hline Once a month or less & 18.6 & $3.5 \mathrm{a}$ & $9.4 \mathrm{a}$ & $36.6_{\mathrm{b}}$ & $47.4_{\mathrm{b}}$ & \multirow{4}{*}{$<0.001$} \\
\hline 2-3 times per month & 28.4 & $16.8 \mathrm{a}$ & $34.1_{\mathrm{b}}$ & $29.3_{\mathrm{a}, \mathrm{b}}$ & $43.4_{\mathrm{b}}$ & \\
\hline 1-2 times per week & 31.6 & $42.0_{a}$ & $38.8_{\mathrm{a}}$ & $26.8 \mathrm{a}$ & $6.6 \mathrm{~b}$ & \\
\hline 3-4 times per week & 14.2 & $23.1_{\mathrm{a}}$ & $12.9 \mathrm{a}, \mathrm{b}$ & $7.3_{\mathrm{b}, \mathrm{c}}$ & $2.6_{c}$ & \\
\hline 5 or more times per week & 7.2 & $14.7_{\mathrm{a}}$ & $4.7_{\mathrm{b}}$ & $0_{\mathrm{b}}$ & $0_{\mathrm{b}}$ & \\
\hline Buys dry beans & 86.8 & $98.7 \mathrm{a}$ & $93.2 \mathrm{~b}$ & $85.4_{\mathrm{b}}$ & $56.6_{c}$ & $<0.001$ \\
\hline \multicolumn{7}{|l|}{$\begin{array}{l}\text { For the } 87 \% \text { who buy dry beans: } \\
\text { Preferred dry bean packaging type }\end{array}$} \\
\hline Loose & 42.5 & $58.8 \mathrm{a}$ & $39.0_{\mathrm{b}}$ & $28.6_{\mathrm{b}}$ & $4.7_{\mathrm{c}}$ & $<0.001$ \\
\hline Small bags & 44.5 & $31.1_{\mathrm{a}}$ & $50.0_{\mathrm{b}}$ & $48.6_{\mathrm{a}, \mathrm{b}}$ & $76.7_{\mathrm{c}}$ & $<0.001$ \\
\hline Medium bags & 23.7 & $22.3_{\mathrm{a}}$ & $20.7 \mathrm{a}$ & $42.9_{\mathrm{b}}$ & $18.6 \mathrm{a}$ & 0.041 \\
\hline Large bags & 10.1 & 13.5 & 9.8 & 0 & 7.0 & n.s. \\
\hline \multicolumn{7}{|l|}{ Important dry bean purchase traits } \\
\hline Price & 58.9 & 59.7 & 52.4 & 54.3 & 72.1 & n.s. \\
\hline Tradition-always buy type & 36.2 & $46.3_{a}$ & $25.6_{\mathrm{b}}$ & $37.1_{\mathrm{a}, \mathrm{b}}$ & $20.9_{\mathrm{b}}$ & 0.002 \\
\hline Quality & 26.2 & 28.9 & 20.7 & 28.6 & 25.6 & n.s. \\
\hline Nutritional value & 25.2 & 26.8 & 28.0 & 14.3 & 23.3 & n.s. \\
\hline Color of beans & 24.6 & 28.9 & 24.4 & 20.0 & 14.0 & n.s. \\
\hline Taste of beans & 20.1 & 18.8 & 14.6 & 25.7 & 30.2 & n.s. \\
\hline Cook quickly & 17.2 & $21.5 \mathrm{a}$ & $11.0_{\mathrm{b}, \mathrm{c}}$ & $5.7_{\mathrm{c}}$ & $23.3_{\mathrm{a}, \mathrm{b}}$ & 0.037 \\
\hline Brand & 14.6 & 17.4 & 12.2 & 11.4 & 11.6 & n.s. \\
\hline \multicolumn{7}{|l|}{$\begin{array}{l}\text { Prefer beans from a specific } \\
\text { country? }\end{array}$} \\
\hline No country preference & 66.1 & $58.8_{a}$ & $67.9_{\mathrm{a}, \mathrm{b}}$ & $80.0_{\mathrm{b}}$ & $76.7_{\mathrm{b}}$ & $<0.001$ \\
\hline Yes, Mexico or Central America & 28.3 & $41.2 \mathrm{a}$ & $28.4_{a}$ & $8.6 \mathrm{~b}$ & $0_{\mathrm{b}}$ & \\
\hline Yes, USA & 5.5 & $0_{a}$ & $3.7_{\mathrm{b}}$ & $11.4_{\mathrm{b}, \mathrm{c}}$ & $23.3_{\mathrm{c}}$ & \\
\hline $\begin{array}{c}\text { Buys canned beans } \\
\text { For the } 50 \% \text { who buy canned beans: } \\
\text { Important canned bean purchase } \\
\text { traits }\end{array}$ & 50.0 & $31.1_{\mathrm{a}}$ & $60.2_{b}$ & $65.9 \mathrm{~b}$ & $67.1_{\mathrm{b}}$ & $<0.001$ \\
\hline Price & 48.9 & $36.2 \mathrm{a}$ & $35.8 \mathrm{a}$ & $40.7 \mathrm{a}$ & $78.4_{\mathrm{b}}$ & $<0.001$ \\
\hline Tradition-always buy type & 20.2 & 21.3 & 18.9 & 37.0 & 11.8 & n.s. \\
\hline Quality & 19.1 & 14.9 & 13.2 & 22.2 & 27.5 & n.s. \\
\hline Nutritional value & 18.5 & 14.9 & 17.0 & 18.5 & 23.5 & n.s. \\
\hline Taste of beans & 41.6 & 34.0 & 41.5 & 48.1 & 45.1 & n.s. \\
\hline Brand & 19.7 & 170 & 15.1 & 22.2 & 25.5 & n.s. \\
\hline
\end{tabular}

Same subscript letters $(\mathrm{a}, \mathrm{b}$, etc.) indicate column proportions that are not significantly different from each other; n.s. $=$ not significant.

Dry and canned purchase patterns were significantly different by acculturation and ethnicity. More of the less acculturated Latinas bought dry beans than any other group $(p<0.001)$. Of the women who purchased dry beans, less acculturated Latinas preferred them loose or in bulk, whereas NHW women bought beans in small 1-2 pound bags ( $p<0.001$ for both). The selection characteristic of tradition-based purchase of dry beans $(p<0.001)$ was more important to less acculturated and more acculturated Latinas than for the bicultural or NHW women. Quick-cooking beans were preferred by less acculturated Latinas and NHW women in contrast to bicultural and more acculturated Latinas $(p=0.037)$. A greater number of less acculturated and bicultural Latinas preferred beans grown in Mexico or Central America than other women $(p<0.001)$.

Of those who reported buying canned beans, less acculturated Latinas purchased them significantly less often when compared to the other three groups $(p<0.001)$. Price was an important factor in canned-bean selection for the NHW women but not as much for all of the Latinas $(p<0.001)$. Twenty-six percent of all women said they did not buy a particular brand, and $13 \%$ stated they did not remember the brand name. About 19\% purchased Rosarita (Conagra, Chicago, IL, USA), 10\% La Costeña (Tucson, 
AZ, USA), 6.5\% Sun Vista (Faribault, MN, USA), and 6\% Goya (Secaucus, NJ, USA) brands. Twenty percent mentioned a wide range of other various brands (data not shown).

Regardless of canned or dry form, women were asked how often they consumed ten nationally popular market classes of pulses [2,7]. Pinto beans were most frequently consumed at least twice per month $(78.3 \%)$, with lentils a distant second $(32.8 \%)$. Black $(25.1 \%)$, baked $(23.9 \%)$, mayocoba $(22.5 \%)$, chickpeas $(19.5 \%)$, navy $(13.3 \%)$, red kidney $(11.7 \%)$, black eyed peas $(7.1 \%)$, and fava beans $(6.4 \%)$ were eaten by fewer women. Pinto, lentil, and mayocoba intakes were significantly higher for the less acculturated and bicultural Latinas in comparison to more acculturated Latinas and NHWs $(p<0.001)$. Chickpea consumption $(p=0.012)$ was significantly lower for the more acculturated Latinas than the other women. Red kidney beans were eaten most frequently by NHWs and rarely by less acculturated Latinas $(p<0.001)$. NHWs and more acculturated Latinas reported higher baked bean consumption than bicultural and less acculturated Latinas $(p=0.001)$, whereas fava bean intakes were mainly among less acculturated Latinas $(p=0.005)$. There were no observed acculturation or ethnic differences for black, navy, or black eyed pea intakes.

The general-bean and canned-bean attitude responses for Likert-type statements are displayed in Table 3. Significant acculturation level/ethnicity related differences were found for 12 of the 14 statements. A greater percentage of Latinas compared to NHW women disagreed that it is difficult to make food with beans $(p=0.005)$, and that they take too long to prepare $(p<0.001)$. While about half of all the women agreed beans can cause intestinal gas, a higher percentage of the less acculturated Latinas than NHW women disagreed with this statement $(p<0.01)$. A larger number of the bicultural and more acculturated Latinas disagreed with the assertion that their family/children refuse to eat beans $(p<0.01)$, and that beans are not eaten by their friends $(p=0.013)$ compared to the less acculturated and the NHW women. When asked about reasons that might prevent them from eating or cooking with canned beans, less acculturated Latinas were more likely to agree that canned beans are not true to their culture, their families will not eat them, they would go without beans if not cooking them themselves, and that canned beans are not healthy, are too expensive, do not taste good, and contain preservatives when compared to the responses from all other groups $(p<0.001$ for all).

Less acculturated Latinas and NHW women had significantly lower or less positive bean attitude scores compared to bicultural and more acculturated Latinas $(p<0.001)$. A general linear model (GLM) tested whether six variables (age, food security score, education level, number of children in household, acculturation category, and bean consumption frequency) could be used to predict a bean-attitude scale. Results supported that $16.4 \%$ of the general bean attitude score variance $\left(R^{2}=0.164 ; \mathrm{F}(22,318)\right.$ $=2.84, p=0.000$; partial $\eta^{2}=0.164$; power $=1.00$ ) was explained by the model. The general bean attitude scale was predicted by bean consumption frequency $\left(\mathrm{F}(4)=3.73 ; p=0.006\right.$; partial $\eta^{2}=0.045$; power $=0.884)$, and acculturation level $\left(\mathrm{F}(3)=3.006 ; p=0.031\right.$; partial $\eta^{2}=0.028$; power $\left.=0.706\right)$.

The average canned-bean attitude scale was significantly lower or less positive for less acculturated Latinas than for any other group $(p<0.001)$. The GLM results for the canned-bean attitude scale indicated $24.6 \%$ of the observed differences in the canned bean-attitude scale was explained by the model $\left(\mathrm{R}^{2}=0.246 ; \mathrm{F}(22,318)=4.720, \mathrm{p}=0.000 ;\right.$ partial $\eta^{2}=0.246$; power $\left.=1.00\right)$. Acculturation level was predictive of canned-bean attitude score $\left(\mathrm{F}(3)=13.89 ; p=0.000\right.$; partial $\eta^{2}=0.116$; power $=1.00$ ). 
Table 3. Beliefs and attitude scores with respect to general and canned beans among low-income Arizona women by Bidimensional Acculturation Scale category $(N=356)$.

\begin{tabular}{|c|c|c|c|c|c|c|}
\hline $\begin{array}{l}\text { Here Are Reasons People Have Said } \\
\text { Prevent Them from Eating or Cooking } \\
\text { Beans. Do You Agree or Disagree? }\end{array}$ & $\begin{array}{c}\text { Total } \\
\text { Sample }\end{array}$ & $\begin{array}{l}\text { Less Acculturated } \\
\text { Latinas } 42 \%(151)\end{array}$ & $\begin{array}{c}\text { Bicultural } \\
\text { Latinas } 25 \%(88)\end{array}$ & $\begin{array}{c}\text { More } \\
\text { Acculturated } \\
\text { Latinas } 12 \%(41)\end{array}$ & $\begin{array}{l}\text { Non-Hispanic } \\
\text { White } 21 \%(76)\end{array}$ & $p$ Value \\
\hline & & $\longleftarrow$ & $\%$ & $\longrightarrow$ & & \\
\hline \multicolumn{7}{|l|}{ 1. Difficult to make food with beans } \\
\hline Disagree & 82.9 & $84.8 \mathrm{a}$ & $89.8_{\mathrm{a}}$ & $87.8_{\mathrm{a}}$ & $68.4_{\mathrm{b}}$ & \multirow{3}{*}{0.005} \\
\hline Neutral & 10.6 & $8.3_{a}$ & $4.5 \mathrm{a}$ & $9.8_{\mathrm{a}, \mathrm{b}}$ & $22.4_{\mathrm{b}}$ & \\
\hline Agree & 6.6 & $6.9 \mathrm{a}$ & $5.7 \mathrm{a}$ & $2.4_{a}$ & $9.2_{\mathrm{a}}$ & \\
\hline \multicolumn{7}{|l|}{ 2. Family/children will not eat } \\
\hline Disagree & 76.6 & $73.3_{\mathrm{a}}$ & $87.5 \mathrm{~b}$ & $90.2_{\mathrm{b}}$ & $63.2 \mathrm{a}$ & \multirow{3}{*}{0.004} \\
\hline Neutral & 10.7 & $11.3_{\mathrm{a}, \mathrm{b}}$ & $6.8 \mathrm{~b}$ & $4.9 \mathrm{a}, \mathrm{b}$ & $17.1_{\mathrm{a}}$ & \\
\hline Agree & 12.7 & $15.3^{a} \mathrm{~V}$ & $5.7_{\mathrm{c}}$ & $4.9_{\mathrm{b}, \mathrm{c}}$ & $19.7_{\mathrm{a}}$ & \\
\hline \multicolumn{7}{|l|}{ 3. Do not like taste of beans } \\
\hline Disagree & 74.7 & 70.9 & 83.0 & 82.9 & 68.4 & \multirow{3}{*}{ n.s. } \\
\hline Neutral & 10.7 & 9.9 & 8.0 & 9.8 & 15.8 & \\
\hline Agree & 14.6 & 19.2 & 9.1 & 7.3 & 15.8 & \\
\hline \multicolumn{7}{|l|}{ 4. Only poor people eat beans } \\
\hline Disagree & 89.0 & 87.3 & 88.6 & 100.0 & 86.8 & \multirow{3}{*}{ n.s. } \\
\hline Neutral & 7.3 & 7.3 & 6.8 & 0 & 11.8 & \\
\hline Agree & 3.7 & 5.3 & 4.5 & 0 & 1.3 & \\
\hline \multicolumn{7}{|l|}{ 5. Beans not eaten by friends } \\
\hline Disagree & 70.5 & $61.6_{a}$ & $78.4_{\mathrm{b}, \mathrm{c}}$ & $87.8_{\mathrm{c}}$ & $69.7_{\mathrm{a}, \mathrm{b}}$ & \multirow{3}{*}{0.013} \\
\hline Neutral & 22.8 & $29.1_{a}^{a}$ & $18.2_{\mathrm{a}, \mathrm{b}}$ & $12.2_{\mathrm{b}}$ & $21.1_{\mathrm{a}, \mathrm{b}}^{\mathrm{b}}$ & \\
\hline Agree & 6.7 & $9.3 \mathrm{a}$ & $3.4_{\mathrm{a}, \mathrm{b}}$ & $0_{\mathrm{b}}$ & $9.2_{\mathrm{a}}$ & \\
\hline \multicolumn{7}{|l|}{ 6. Beans cause intestinal gas } \\
\hline Disagree & 21.4 & $30.5 \mathrm{a}$ & $19.3_{\mathrm{a}, \mathrm{b}}$ & $15.0_{a, b}$ & $9.2 \mathrm{~b}$ & \multirow{3}{*}{0.003} \\
\hline Neutral & 32.1 & $24.5 \mathrm{a}$ & $30.7_{\mathrm{a}, \mathrm{b}}$ & $45.0_{\mathrm{b}}$ & $42.1_{\mathrm{b}}$ & \\
\hline Agree & 46.5 & $45.0_{\mathrm{a}}$ & $50.0_{\mathrm{a}}$ & $40.0_{\mathrm{a}}$ & $48.7 \mathrm{a}$ & \\
\hline \multicolumn{7}{|l|}{ 7. Dry beans take too long to prepare } \\
\hline Disagree & 54.4 & $64.9 \mathrm{a}$ & $52.9 \mathrm{a}$ & $58.5 \mathrm{a}$ & $32.9_{\mathrm{b}}$ & \multirow{3}{*}{$<0.001$} \\
\hline Neutral & 17.7 & $14.6_{a}^{a}$ & $16.1_{\mathrm{a}}$ & $22.0_{a}$ & $23 . \mathrm{a}_{\mathrm{a}}$ & \\
\hline Agree & 27.9 & $20.5 \mathrm{a}$ & $31.0_{\mathrm{a}, \mathrm{b}}$ & $19.5 \mathrm{a}$ & $43.4_{\mathrm{b}}$ & \\
\hline \multicolumn{7}{|l|}{ General bean attitude scale } \\
\hline $\begin{array}{c}(\mu \pm \mathrm{SD}) \\
\text { (Summary of questions } 1-5+\text { ) }\end{array}$ & $20.7 \pm 3.7$ & $20.2 \pm 3.6$ & $21.7 \pm 3.3$ & $22.3 \pm 2.6$ & $19.6 \pm 4.0$ & 0.001 \\
\hline 8. Canned beans not true to culture & & & & & & \\
\hline Disagree & 74.4 & $60.7 \mathrm{a}$ & $81.4_{\mathrm{b}}$ & $97.6_{\mathrm{c}}$ & $80.3_{\mathrm{b}}$ & $<0001$ \\
\hline Neutral & 15.5 & $21.4_{a}$ & $9.3_{\mathrm{b}, \mathrm{c}}$ & $2.4_{\mathrm{c}}$ & $18.4_{\mathrm{a}, \mathrm{b}}$ & $<0.001$ \\
\hline Agree & 10.1 & $17.9 \mathrm{a}$ & $9.3 \mathrm{a}$ & $0_{\mathrm{b}}$ & $1.3_{\mathrm{b}}$ & \\
\hline 9. Family will not eat canned beans & & & & & & \\
\hline Disagree & 56.8 & $39.1_{a}$ & $65.5_{\mathrm{b}}$ & $77.5_{\mathrm{b}}$ & $71.1_{\mathrm{b}}$ & \\
\hline Neutral & 16.7 & $18.5 \mathrm{a}$ & $14.9 \mathrm{a}$ & $12.5 \mathrm{a}$ & $17.1_{\mathrm{a}}$ & $<0.001$ \\
\hline Agree & 26.6 & $42.4_{a}$ & $19.5 \mathrm{~b}$ & $10.0_{\mathrm{b}}$ & $11.8_{\mathrm{b}}$ & \\
\hline 10. If I cannot cook beans myself will & & & & & & \\
\hline go without & & & & & & \\
\hline Disagree & 66.2 & $60.7 \mathrm{a}$ & $72.4_{\mathrm{a}, \mathrm{b}}$ & $78.0_{\mathrm{b}}$ & $63.2_{\mathrm{a}, \mathrm{b}}$ & $<0.001$ \\
\hline Neutral & 18.6 & $14.5 \mathrm{a}$ & $16.1_{\mathrm{a}}$ & $14.6_{a}$ & $31.6_{\mathrm{b}}$ & \\
\hline Agree & 15.2 & $24.8 \mathrm{a}$ & $11.5_{\mathrm{b}}$ & $7.3_{\mathrm{b}}$ & $5.3_{\mathrm{b}}$ & \\
\hline 11. Canned beans are not healthy & & & & & & \\
\hline Disagree & 52.2 & $31.5 \mathrm{a}$ & $55.2_{\mathrm{b}}$ & $75.6_{\mathrm{c}}$ & $75.0_{\mathrm{c}}$ & \\
\hline Neutral & 32.6 & $42.0_{a}$ & $32.2_{\mathrm{a}, \mathrm{b}}$ & $22.0_{\mathrm{b}}$ & $21.1_{\mathrm{b}}$ & $<0.001$ \\
\hline Agree & 15.3 & $26.6 \mathrm{a}$ & $12.6_{\mathrm{b}}$ & $2.4_{\mathrm{b}, \mathrm{c}}$ & $3.9 \mathrm{c}$ & \\
\hline 12. Canned beans are too expensive & & & & & & \\
\hline Disagree & 58.7 & $41.8_{\mathrm{a}}$ & $67.4_{\mathrm{b}}$ & $68.3_{\mathrm{b}}$ & $76.3_{\mathrm{b}}$ & \\
\hline Neutral & 24.1 & $32.2_{a}^{a}$ & $19.8_{\mathrm{b}}$ & $17.1_{\mathrm{a}}$ & $17.1_{\mathrm{b}}$ & $<0.001$ \\
\hline Agree & 17.2 & $26 . a_{a}$ & $12.8_{\mathrm{b}}$ & $14.6_{\mathrm{a}}$ & $6.6_{\mathrm{b}}$ & \\
\hline 13. Canned beans do not taste good & & & & & & \\
\hline Disagree & 47.8 & $32.5 \mathrm{a}$ & $52.3_{\mathrm{b}}$ & $61.0_{\mathrm{b}}$ & $65.8_{\mathrm{b}}$ & 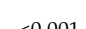 \\
\hline Neutral & 19.4 & $19.2_{\mathrm{a}}$ & $19.3_{a}$ & $19.5_{\mathrm{a}}$ & $19.7 \mathrm{a}$ & $<0.001$ \\
\hline Agree & 32.9 & $48.3_{a}$ & $28.4_{\mathrm{b}}$ & $19.5 \mathrm{~b}$ & $14.5_{\mathrm{c}}$ & \\
\hline 14. Canned beans have preservatives & & & & & & \\
\hline Disagree & 23.5 & $16.9 \mathrm{a}$ & $27.1_{\mathrm{a}, \mathrm{b}}$ & $39.0_{\mathrm{b}}$ & $23.7_{\mathrm{a}, \mathrm{b}}$ & \\
\hline Neutral & 30.5 & $19.7 \mathrm{a}$ & $29.4_{a, b}$ & $39.0_{\mathrm{b}, \mathrm{c}}$ & $47.4_{\mathrm{C}}$ & $<0.001$ \\
\hline Agree & 45.9 & $63.4_{a}$ & $43.5_{\mathrm{b}}$ & $22.0_{\mathrm{c}}$ & $28.9_{\mathrm{b}, \mathrm{c}}$ & \\
\hline $\begin{array}{l}\text { Canned bean attitude scale } \\
\quad(\mu \pm \text { SD) } \\
\text { (Summary of questions } 8-13+\text { ) }\end{array}$ & $21.7 \pm 4.9$ & $19.2 \pm 4.8$ & $22.8 \pm 5.0$ & $24.4 \pm 3.9$ & $23.8 \pm 3.8$ & $<0.001$ \\
\hline
\end{tabular}

t Items reverse coded for summation. Same subscript letters ( $a, b$, etc.) indicate column proportions that are not significantly different from each other.

\section{Discussion}

The findings support the hypothesis that dry and canned bean consumption, purchasing preferences, and attitude scores vary by acculturation status and ethnicity among low-income Latinas and NHW women. Differences in variable values corresponded to categorical acculturation levels progressing from less acculturated to bicultural to more acculturated Latinas with respect to consumption frequencies, purchasing preferences, knowledge of preparation, and attitudes towards 
beans and canned beans. These trends are consistent with other studies showing more acculturated US Latinos, notably Mexican Americans, as well as Puerto Ricans, are eating fewer beans over time $[5,14,19]$. However, the current study is one of a few with a large enough sample size to characterize the nuances of acculturation and bean consumption patterns.

The most positive general-bean attitude scores were in the bicultural and more acculturated Latinas rather than in the less acculturated group, suggesting beans remain a strong marker of Latino cultural identity independent of reported consumption, as observed in additional studies [5,14]. The positive general-bean attitude scores may be due to exposure of healthy bean messaging in other mainstream media programs not received by less acculturated Latinas. In any case, such positive attitudes among "acculturating" women may serve as a leverage point for achieving greater consumption through appropriate messaging that contributes to the cultural and nutritional value of beans and other pulses [5,19]. As part of the nutrition transition even within Mexico, a recent study found positive attitudes about legumes for their taste, health benefits, and nutrition, despite consumption levels lower than recommendations [35].

Based on previous research, Hispanic women were expected to agree that canned beans were not true to culture [26]. However, the survey indicated that most disagree with this assertion. The minimal use of canned beans by less acculturated Latinas may be due to reasons other than cultural discrimination alone. Concerns about preservatives, health, price, family acceptance, taste, a general reluctance to use canned or ready-made products, or simply a greater preference for dry beans are alternate explanations. Qualitative research could help discern reasons behind the apparent negative attitude toward canned beans to guide nutrition education approaches [36].

Some women in the current study expressed concern about use of preservatives in canned beans. Park et al. found that Latinas defined healthy foods in terms of purity and freshness, which are qualities typically associated with foods eaten in their countries of origin [37]. Alternatively, Ramirez et al. found that bicultural Hispanic women in California perceived American foods as healthier than some traditional Mexican foods [38]. Lack of access to preferred bean types, forms (dry and canned), and packaging in areas of the US may contribute to reduced bean consumption. Availability of desired ingredients was the most common reason preventing Hispanic women living in Belgium from cooking their traditional cuisine [39]. However, ingredient shortage is unlikely in the American Southwest as compared to Latinas in Iowa or New England [15,19].

For consumers interested in natural, non-genetically modified, or gluten free products, canned or dry beans are minimally processed and are a nutrient-rich, easily accessible, and affordable food that should be recommended as part of a healthy diet $[7,10]$. A recent survey exploring open-ended definitions of healthy foods found similar desires for less processed, low fat, and high in nutrients between Hispanics and non-Hispanics [40]. While the general public often equates "freshness" with nutrition or health, research shows that similar nutrient profiles are delivered by canned fruits and vegetables, and dry or canned beans [41]. Education about health-related and nutritional similarities between dry and canned beans may be beneficial to dispel misconceptions about canned foods that may inhibit usage and contribute to nutrition inadequacies [42]. Canned foods also last longer than fresh, which can increase food and nutrition security for individuals seeking to economically increase nutritional quality [41].

Although price was an important factor in dry or canned bean selection for all women, Palmer et al. found that designating beans as 'cheap' could dissuade rather than encourage low-income women in Iowa from purchase, suggesting an emphasis on value, tradition, or health benefits might be better themes of promotion [42]. Because less acculturated Latinas in this study prefer dry over canned beans and are proficient in preparing dry beans, program materials may want to utilize their skills and focus on supporting this preference. Programming with an emphasis on the equivalent nutrition elements of canned beans with dry beans could encourage Latinas and NHW women to retain beans in their diets with less perceived preparation time $[16,43,44]$. Education on using dry beans could benefit NHW women who had less knowledge of how to prepare dry beans, but were also concerned with the price 
of canned. Nutrition education should also address concerns that eating beans will cause intestinal gas or flatulence. Increased flatulence has been suggested to be a transitory effect, or based more on preconceptions. Advice on adding small amounts of beans gradually into the diet could encourage wary consumers to try them until the gut adjusts $[45,46]$.

To counteract acculturation-related decline in bean consumption, culturally sensitive programs should encourage and support retention of traditional healthy eating patterns by low-income Hispanic women. Such programs have been shown to yield better knowledge and health outcomes using healthy adaptations of favorite Mexican American recipes [47]. Emphasis on positive cultural practices may result in more meaningful and attainable health recommendations for immigrants $[17,21]$. Integration of cultural identities has also led to lower levels of acculturation-related stress and better psychological functioning [48,49]. This study's findings can be used to appropriately target low-income Latinas based on bean-related purchasing preferences and attitudes in the metro-Phoenix, Arizona region.

This research has several strengths, including addressing the gap in knowledge of dry and canned bean purchasing preferences, bean consumption patterns, and attitudes among low-income Latinas and NHW women in the Southwest. The observed changes in a cultural dietary element like beans help us to better understand the nuances of dietary acculturation among Latinas which appears to be more complex than simply linear $[1,4,5]$. Additionally, less research has been conducted with female EFNEP participants as compared to women utilizing other food assistance and education programs like WIC, or SNAP. To reduce sampling bias toward bean consumers only, recruitment materials asked about general eating patterns without mentioning beans. Through the collaborative community relationships established with EFNEP and WIC, data collection was facilitated during immigration-related political unrest in Arizona. The insights into the bean views of monolingual Spanish-speaking Latinas who trusted the field staff enough to participate are a unique study strength [50].

Several limitations are to be noted in the study. The data collected were from a convenience sample of mostly Mexican origin Latinas and NHW women who were eligible for government assistance programs. As a non-random sample, these results should not be extrapolated to low-income women from other geographic areas, ethnicities, races, ages, or non-program participants. Since data are cross-sectional, the influence of acculturation processes over time cannot be evaluated. Respondents were not asked if beans were used as a main meal, side dish, or meat substitute, the serving size consumed, nor how beans were prepared. Some of the observed differences between groups by acculturation and ethnicity should be explored through qualitative methods such as interviews or focus groups to better understand the context and meaning of behaviors.

\section{Conclusions}

The results of this study suggest ways to tailor nutrition messages about beans and other pulses based on preferences, attitudes, and purchasing behaviors of low-income Latinas and NHW women in the Southwest US. Further research on food beliefs, preferences, practices, variety and manner of bean use, knowledge of how beans are incorporated into daily diets, and perceptions of canned foods would provide additional insight to aid in the promotion of beans and other pulses to low-income women. A deeper understanding of how acculturation affects bean consumption can direct culturally sensitive programing toward greater use of these beneficial components of traditional Latino diets. Identifying underlying influences on choices by low-income women regarding bean consumption could lead to better-informed programming to meet US DGA recommendations for maintaining overall health and preventing chronic disease.

Author Contributions: Funding acquisition and supervision, D.M.W.; Study Design, D.M.W.; Participant recruitment, screening, and data collection, D.M.W.; Data and statistical analysis, D.M.W., and M.M.H.; Writing-Original draft preparation, M.M.H.; Writing—Review and Editing, M.M.H. and D.M.W.; Writing-Final Review and Approval, M.M.H. and D.M.W. All authors have read and agreed to the published version of the manuscript. 
Funding: Partial funding for original data collection was provided by a grant from the US Dry Bean Council to D.M.W. Research support for analysis and manuscript preparation for M.M.H. and D.M.W. was provided by the Iowa Agriculture and Home Economics Experiment Station, Ames, Iowa. Project No. IOW05462 is sponsored by the Hatch Act and State of Iowa funds.

Conflicts of Interest: The authors declare no conflict of interest. The funders had no role in the design of the study; in the collection, analyses, or interpretation of data; in the writing of the manuscript, or in the decision to publish the results.

\section{References}

1. Amerlinck, M.J. Rice and beans, a staple on even the most respectable Mexican tables. In Rice and Beans: A Unique Dish in a Hundred Places; Wilk, R., Barbosa, L., Eds.; Berg Publishers: New York, NY, USA, 2012; Volume 1, pp. 219-240.

2. Zahniser, S.; Torres, M.V.; Cuellar, J.A.; Alvarez, J.A.; Lopez, N.F.; Bhatta, R. The US and Mexican Dry Bean Sectors. In VGS-341-01, USDA/ERS, December 2010; Diane Publishing: Collingdale, PA, USA, 2011.

3. Ayala, G.X.; Baquero, B.; Klinger, S. A systematic review of the relationship between acculturation and diet among Latinos in the United States: Implications for future research. J. Am. Diet Assoc. 2008, 108, 1330-1344. [CrossRef]

4. Satia-Abouta, J.; Patterson, R.E.; Neuhouser, M.L.; Elder, J. Dietary acculturation: Applications to nutrition research and dietetics. J. Am. Diet Assoc. 2002, 102, 1105-1118. [CrossRef]

5. Smith, W.E.; Day, R.S.; Brown, L.B. Heritage retention and bean intake correlates to dietary fiber intakes in Hispanic mothers-Qué Sabrosa Vida. J. Am. Diet. Assoc. 2005, 105, 404-411. [CrossRef]

6. Hiza, H.A.; Casavale, K.O.; Guenther, P.M.; Davis, C.A. Diet quality of Americans differs by age, sex, race/ethnicity, income, and education level. J. Acad. Nutr. Diet. 2013, 113, 297-306. [CrossRef]

7. Mitchell, D.C.; Lawrence, F.R.; Hartman, T.J.; Curran, J.M. Consumption of dry beans, peas, and lentils could improve diet quality in the US population. J. Am. Diet Assoc. 2009, 109, 909-913. [CrossRef]

8. Batis, C.; Hernandez-Barrera, L.; Barquera, S.; Rivera, J.A.; Popkin, B.M. Food acculturation drives dietary differences among Mexicans, Mexican Americans, and non-Hispanic whites. J. Nutr. 2011, 141, 1898-1906. [CrossRef]

9. Jaimes, N.; Londono, V.; Halpern, A.C. The term Hispanic/Latino: A note of caution. JAMA Dermatol. 2013, 149, 274-275. [CrossRef]

10. Dietary Guidelines 2015-2020: A Closer Look Inside Healthier Eating Patterns. Health gov Web Site. Available online: https://health.gov/dietaryguidelines/2015/guidelines/chapter-1/a-closer-look-inside-healthy-eatingpatterns/\#callout-legumes (accessed on 21 June 2018).

11. Havemeier, S.; Erickson, J.; Slavin, J. Dietary guidance for pulses: The challenge and opportunity to be part of both the vegetable and protein food groups. Ann. N. Y. Acad. Sci. 2017, 1392, 58-66. [CrossRef]

12. Food and Agriculture Organization of the United Nations. Pulses: Nutritious Seeds for a Sustainable Future. 2016. Rome, Italy. Available online: http://www.fao.org/3/a-i5528e.pdf (accessed on 16 March 2020).

13. Thorn, B.; Huret, N.; Bellows, D.; Ayo, E.; Myers, R.; Wilcox-Cook, E. WIC Food Packages Policy Options Study II; Food and Nutrition Service, US Dept. of Agriculture: Alexandria, VA, USA, 2015.

14. United States Department of Agriculture Food and Nutrition Service. Programs and Services. Available online: https://www.fns.usda.gov/programs-and-services (accessed on 22 June 2018).

15. Winham, D.M.; Tisue, M.E.; Palmer, S.M.; Cichy, K.A.; Shelley, M.C. Dry bean preferences and attitudes among Midwest Hispanic and Non-Hispanic white women. Nutrients 2019, 11, 178. [CrossRef]

16. Radford, A.; Dahl, W.J. Identifying learning needs of WIC participants regarding dry beans. J. NEAFC 2014, 9,63-69.

17. McClain, A.C.; Dickin, K.L.; Dollahite, J. Life course influences on food provisioning among low-income, Mexican-born mothers with young children at risk of food insecurity. Appetite 2019, 132, 8-17. [CrossRef] [PubMed]

18. Zamora, M.; Bernsten, R. Assessing markets for Central American products in US ethnic communities (The Common Beans Case). Rev. Latinoam. Desarro. Económico 2005, 4, 111-142.

19. Mattei, J.; Campos, H. Perceptions and behaviors of legume consumption among Puerto Rican adults. Health Behav. Policy Rev. 2014, 1, 38-49. [CrossRef] 
20. Wiesinger, J.; Cichy, K.; Tako, E.; Glahn, R. The fast cooking and enhanced iron bioavailability properties of the Manteca yellow bean (Phaseolus vulgaris L.). Nutrients 2018, 10, 1609. [CrossRef]

21. Gans, K.M.; Risica, P.M.; Strolla, L.O.; Fournier, L.; Kirtania, U.; Upegui, D.; Zhao, J.; George, T.; Acharyya, S. Effectiveness of different methods for delivering tailored nutrition education to low income, ethnically diverse adults. Int. J. Behav. Nutr. Phys. Act. 2009, 6, 24. [CrossRef]

22. Winham, D.M.; Szkupinski Quiroga, S.; Underiner, T.L.; Etheridge Woodson, S.; Todd, M.A. Integration of theatre activities in cooking workshops improves healthy eating attitudes among ethnically diverse adolescents: A pilot study. ICAN Infant Child Adolesc. Nutr. 2014, 6, 99-108. [CrossRef]

23. Motel, S.; Patten, E. 2011, Foreign-Born Population in the United States Statistical Portrait. Pew. Research Center-Hispanic Trends. Available online: http://www.pewhispanic.org/2012/02/21/2010-statisticalinformation-on-immigrants-in-united-states/ (accessed on 12 November 2019).

24. Arizona Department of Health Services. WIC Needs Assessment. Az.gov Website. Available online: http: //www.azdhs.gov/azwic/documents/local_agencies/reports/wic-needs-assessment-02-22-13.pdf (accessed on 15 March 2019).

25. Maricopa County Cooperative Extension, University of Arizona. Maricopa County Cooperative Extension, University of Arizona. Maricopa County Summary of Adult Participant Profiles 2011. In Unit AZ013-A; Maricopa County Cooperative Extension: Phoenix, AZ, USA, 2012.

26. Winham, D.M.; Armstrong Florian, T.L. Hispanic women in EFNEP have low adherence with dietary guidelines regardless of acculturation level. J. Hunger. Environ. Nutr. 2010, 5, 498-509. [CrossRef]

27. Winham, D.M.; Armstrong Florian, T.L.; Thompson, S.V. Low-income US women under-informed of the specific health benefits of consuming beans. PLoS ONE 2016, 11, e0147592. [CrossRef]

28. Winham, D.M.; Armstrong Florian, T.L. Nativity, not acculturation, predicts SNAP usage among low-income Hispanics with food insecurity. J. Hunger. Environ. Nutr. 2015, 10, 202-213. [CrossRef]

29. Winham, D.M.; Palmer, S.M.; Armstrong Florian, T.L.; Shelley, M.C. Health behaviors among low-income Hispanic and non-Hispanic white women. Am. J. Health Behav. 2018, 42, 56-68. [CrossRef]

30. Marin, G.; Gamba, R.J. A new measurement of acculturation for Hispanics: The Bidimensional Acculturation Scale for Hispanics (BAS). Hisp. J. Behav. Sci. 1996, 18, 297-316. [CrossRef]

31. Purdue University. Expanded Food and Nutrition Education Program Food Recall at Entry. 2012. Available online: https://www2.ag.purdue.edu/programs/hhs/efnep/Resource/Adult\%20Enrollment.pdf (accessed on 5 November 2016).

32. Core Food Security Module U.S. Household Food Security Survey Module: Six-Item Short Form, Economic Research Service, USDA, September 2012. Available online: https://www.ers.usda.gov/media/8271/hh2012. pdf (accessed on 13 July 2018).

33. Harrison, G.G.; Stormer, A.; Herman, D.R.; Winham, D.M. Development of a Spanish-language version of the US household food security survey module. J. Nutr. 2003, 133, 1192-1197. [CrossRef] [PubMed]

34. Field, A. Discovering Statistics Using IBM SPSS Statistics; Sage Publications: Thousand Oaks, CA, USA, 2013.

35. Monge, A.; Macias, L.; Campos, H.; Lajous, M.; Mattei, J. Perceptions and reasons for legume consumption in Mexico. Nutr. Food Sci. 2019, 49, 1232-1242. [CrossRef]

36. Kegler, M.C.; Raskind, I.G.; Comeau, D.L.; Griffith, D.M.; Cooper, H.L.; Shelton, R.C. Study design and use of inquiry frameworks in qualitative research published in health education \& behavior. Health Educ. Behav. 2019, 46, 24-31.

37. Park, Y.; Quinn, J.; Florez, K.; Jacobson, J.; Neckerman, K.; Rundle, A. Hispanic immigrant women's perspective on healthy foods and the New York City retail food environment: A mixed-method study. Soc. Sci. Med. 2011, 73, 13-21. [CrossRef]

38. Ramírez, A.S.; Golash-Boza, T.; Unger, J.B.; Baezconde-Garbanati, L. Questioning the dietary acculturation paradox: A mixed-methods study of the relationship between food and ethnic identity in a group of Mexican-American women. J. Acad. Nutr. Diet. 2018, 118, 431-439. [CrossRef]

39. Verbeke, W.; Poquiviqui, G.L. Ethnic food attitudes and behaviour among Belgians and Hispanics living in Belgium. Br. Food J. 2005, 107, 823-840. [CrossRef]

40. Palmer, S.M.; Winham, D.M. Midwest consumer shopping habits, nutrition knowledge, and Latino tienda use. Health Behav. Policy Rev. 2020, 7, 79-91. [CrossRef]

41. Comerford, K. Frequent canned food use is positively associated with nutrient-dense food group consumption and higher nutrient intakes in US children and adults. Nutrients 2015, 7, 5586-5600. [CrossRef] 
42. Palmer, S.; Winham, D.; Oberhauser, A.; Litchfield, R. Socio-ecological barriers to dry grain pulse consumption among low-income women: A mixed methods approach. Nutrients 2018, 10, 1108. [CrossRef]

43. Karlsen, M.C.; Pollard, K.J. Strategies for practitioners to support patients in plant-based eating. J. Geriatr. Cardiol. 2017, 14, 338.

44. Desrochers, N.; Brauer, P.M. Legume promotion in counselling: An e-mail survey of dietitians. Can. J. Diet. Pract. Res. 2001, 62, 193.

45. O'Donnell, A.U.; Fleming, S.E. Influence of frequent and long-term consumption of legume seeds on excretion of intestinal gases. Am. J. Clin. Nutr. 1984, 40, 48-57. [CrossRef]

46. Dahl, W.J.; Hanifi, A.; Zello, G.A.; Tyler, R.T. Gastrointestinal tolerance to daily canned chickpea intake. Can. J. Diet. Pract. Res. 2014, 75, 218-221. [CrossRef]

47. Brown, S.A.; Garcia, A.A.; Kouzekanani, K.; Hanis, C.L. Culturally competent diabetes self-management education for Mexican Americans: The Starr County border health initiative. Diabetes Care 2002, 25, 259-268. [CrossRef]

48. Chen, S.X.; Benet-Martínez, V.; Harris, M.B. Bicultural Identity, bilingualism, and psychological adjustment in multicultural societies: Immigration-based and globalization-based acculturation. J. Personal. 2008, 76, 803-838. [CrossRef]

49. López, I.R.; Contreras, J.M. The best of both worlds: Biculturality, acculturation, and adjustment among young mainland Puerto Rican mothers. J. Cross-Cult. Psychol. 2005, 36, 192-208. [CrossRef]

50. Szkupinski Quiroga, S.A. Vamos a aguantar: Observations on how Arizona's SB 1070 has affected one community. Lat. Stud. 2013, 11, 580-586. [CrossRef]

(C) 2020 by the authors. Licensee MDPI, Basel, Switzerland. This article is an open access article distributed under the terms and conditions of the Creative Commons Attribution (CC BY) license (http://creativecommons.org/licenses/by/4.0/). 